\title{
Clinical utility and cost analysis of routine postoperative head CT in elective aneurysm clippings
}

\author{
Corinna C. Zygourakis, MD, ${ }^{1}$ Ethan Winkler, MD, PhD, ${ }^{1}$ Lawrence Pitts, MD, ${ }^{1}$ Lisa Hannegan, MS, ${ }^{1}$ \\ Benjamin Franc, MD, ${ }^{2}$ and Michael T. Lawton, MD $^{1}$
}

Departments of ${ }^{1}$ Neurological Surgery and ${ }^{2}$ Radiology, University of California, San Francisco, California

\begin{abstract}
OBJECTIVE Postoperative head CT scanning is performed routinely at the authors' institution on all neurosurgical patients after elective aneurysm clippings. The goal of this study was to determine how often these scans influence medical management and to quantify the associated imaging costs.

METHODS The authors reviewed the medical records and accounting database of 304 patients who underwent elective (i.e., nonruptured) aneurysm clipping performed by 1 surgeon (M.T.L.) from 2010 to 2014 at the University of California, San Francisco. Specifically, the total number of postoperative head CT scans, radiographic findings, and the effect of these studies on patient management were determined. The authors obtained the total hospital costs for these patients, including the cost of imaging studies, from the hospital accounting database.

RESULTS Overall, postoperative CT findings influenced clinical management in $3.6 \%$ of cases; specifically, they led to permissive hypertension in 4 patients for possible ischemia, administration of mannitol for edema and high-flow oxygen for pneumocephalus in 2 patients each, seizure prophylaxis in 1 patient, Plavix readjustment in 1 patient, and return to the operating room for an asymptomatic epidural hematoma evacuation in 1 patient. When patients were stratified on the basis of postoperative neurological examination, findings on CT scans altered management in $1.1 \%, 4.8 \%$, and $9.0 \%$ of patients with no new neurological deficits, a nonfocal examination, and focal deficits, respectively. The mean total hospital cost for treating patients who undergo elective aneurysm clipping was $\$ 72,227( \pm \$ 53,966)$ (all values are US dollars), and the cost of obtaining a noncontrast head CT scan was $\$ 292$. Neurologically intact patients required 99 head CT scans, at a cost of $\$ 28,908$, to obtain 1 head CT scan that influenced medical management. In contrast, patients with a focal neurological deficit required only 11 head CT scans, at a cost of $\$ 3212$, to obtain 1 head CT scan that changed clinical management.
\end{abstract}

CONCLUSIONS Although there are no clear guidelines, the large number and high cost of CT scans needed to treat neurologically intact elective aneurysm patients suggest that careful neurological monitoring may be more clinically useful and a better use of hospital resources than routine postoperative CT.

http://thejns.org/doi/abs/10.3171/2016.1.JNS152242

KEY WORDS head CT; CT utilization; aneurysm surgery; cost analysis; vascular disorders

$\mathrm{T}$ He use of CT is extremely common, with an estimated 70 million scans performed annually in the United States. ${ }^{4}$ At our institution, neurosurgeons order routine head CT scans after many surgical procedures, including after all elective (i.e., nonruptured) aneurysm clippings. The performance of head CT scanning is costly, ranging in price from approximately $\$ 200$ to more than $\$ 5000$ at different locations in the United States, ${ }^{7}$ and it is estimated that each head CT scan leads to a $0.07 \%$ increased lifetime risk of cancer as a result of radiation exposure. ${ }^{3}$ Despite the high prevalence, cost, and potential risk, the clinical efficacy of routine head CT has not been proven in many clinical settings.

In a comprehensive literature search, we found several studies addressing the utility of head CT in trauma, $, 2,5,13,17,18$ atraumatic headaches, ${ }^{15}$ and pediatric patients, ${ }^{8,14,16}$ but only 2 studies addressed the clinical utility of routine head CT use after neurosurgical procedures. ${ }^{9,10}$ One study by Fontes et al. examined the clinical utility of postoperative head CT in 892 patients after intracranial neurosurgical procedures. In patients with neurological examination changes or who were comatose postoperatively, postopera-

ABBREVIATIONS CTPA = CT pulmonary angiography; ICU = intensive care unit; $m S v=$ milliseivert; $O R=$ operating room; UCSF = University of California, San Francisco. SUBMITTED September 23, 2015. ACCEPTED January 15, 2016.

INCLUDE WHEN CITING Published online April 29, 2016; DOI: 10.3171/2016.1.JNS152242. 
tive head CT provided positive findings that led to nonsurgical or surgical interventions in anywhere from $10 \%$ to $22 \%$ of cases. In patients with stable neurological examinations, however, CT led to nonsurgical interventions in only $2.2 \%-2.4 \%$ of cases, and no patients required emergency surgical intervention based on a postoperative head CT finding. ${ }^{9}$ Another group found a similarly low yield of findings by postoperative head $\mathrm{CT}$ after neurosurgical procedures. In their analysis of 126 postoperative head CT scans ordered over a 5-month period at the University of California, Los Angeles (UCLA), neurosurgery service, they found only 6 scans with positive findings and 1 scan $(<1 \%)$ that changed management. ${ }^{10}$

To the best of our knowledge, although there are a few recent studies looking at the clinical utility of postoperative head CT, ${ }^{9,10}$ there are no studies addressing the cost of postoperative head CT in neurosurgical patients. The goal of our study was, therefore, to determine both the clinical utility and the cost of obtaining routine postoperative head CT scans in our elective aneurysm clipping patients at the University of California, San Francisco (UCSF).

\section{Methods}

Following study approval by our institutional review board, we searched the UCSF radiology database for all head CT scans performed from 2010 to 2014. From these, we identified which scans were noncontrast and which ones were ordered by neurosurgical providers (i.e., neurosurgery attending physicians, residents, or nurse practitioners).

We then searched the UCSF neurosurgery database for all patients who underwent surgical clipping of nonruptured aneurysms by a specific UCSF cerebrovascular surgeon (M.T.L.) between 2010 and 2014. These were consecutively treated patients, and no patients were excluded from the study. This time frame was selected because it is the period for which we have accessible UCSF cost data. For each patient, we carefully reviewed the medical records to determine the number of postoperative head CT scans obtained, radiographic findings, postoperative neurological examination results, and if/how the postoperative head CT changed patient management. For clinical management changes, we considered the following categories: return to the operating room $(\mathrm{OR})$, placement of an intracranial pressure monitor, blood pressure change, administration of mannitol, administration of high-flow oxygen, lying the patient flat, or other changes. Preoperative and postoperative notes, as well as patient orders, were carefully reviewed. A change in management was considered to be the result of postoperative CT if explicitly stated in the progress note or if there was a clear temporal relationship between completion of the CT scan and/or reporting of the CT results and the completion of new orders in the electronic medical record. Because we hypothesized that we would find little impact of head CT on clinical management, we wanted to ensure that we did not miss any potential cases where CT contributed to changed clinical management. As a result, for all cases, if we were uncertain about whether a management change was attributable to the imaging, we chose to attribute the change to the head CT.
Cost data were obtained as US dollar values from the UCSF hospital cost accounting database for our selected patient cohort. Total costs (direct and indirect, as calculated in the UCSF financial accounting databases) were obtained for each separate patient encounter (i.e., hospitalization and office visit) and summed for each patient. The intent was to capture the entire cost of care associated with the cerebral aneurysm and not just the index hospitalization. Means are expressed as $\pm \mathrm{SD}$. Of note, physician payments and non-UCSF costs incurred in the care of a patient with a cerebral aneurysm (e.g., inpatient rehabilitation stay after discharge and outpatient physical therapy) are not available in our database and, therefore, are not included in our calculations.

Data collection and basic manipulations were performed in Microsoft Excel. JMP version 12 (SAS) was used to perform univariate statistical analyses. A mixeddirection stepwise regression model was then run to determine the variables most predictive of a CT scan leading to a clinical management change.

\section{Results}

Neurosurgical providers ordered 10,677 head CT scans, or $27 \%$ of all noncontrast head CT scans performed at our institution, from January 2010 to December 2014 (Fig. 1). In this study, we focus on the 473 head CT scans that were obtained for our nonruptured aneurysm patients. These represent $4.4 \%$ of all head CT scans ordered by neurosurgical providers at our institution.

Table 1 summarizes our patient cohort characteristics. In our 304 consecutively treated nonruptured aneurysm patients, the mean number of CT scans per patient was $1.54 \pm 1.0$, and the total number of CT scans for these patients was 473 . Every patient underwent at least 1 postoperative noncontrast head CT scan, usually within 6 hours of surgery, although 2 patients received 7 head CT scans each during the course of their hospitalizations. Approximately $20 \%$ of patients also underwent MRI during their hospitalization (mean number of MRI studies performed per patient, $0.20 \pm 0.50$ ). In addition, $85 \%$ of patients underwent cerebral angiography (mean number of angiographic studies per patient, $0.84 \pm 0.45$; Table 1$)$. The majority of patients were neurologically intact or at their neurological preoperative baseline (56\%) on their immediate postoperative neurological examination the night after surgery, although $14 \%$ of patients had nonfocal neurological deficits (usually disorientation) and $26 \%$ had focal neurological deficits (e.g., focal weakness and cranial nerve deficit; Table 1).

Overall, postoperative CT findings influenced clinical management in 11 of 304 patients, or $3.6 \%$ of cases. Specifically, head CT results likely led to permissive hypertension in 4 patients for possible ischemia, administration of mannitol for edema and high-flow oxygen for pneumocephalus in 2 patients each, seizure prophylaxis in 1 patient, Plavix readjustment in 1 patient, and return to the OR for an asymptomatic epidural hematoma evacuation in 1 patient (Table 2).

In patients with focal neurological deficits, head CT led to management changes in $9.0 \%$ of cases (Fig. 2). In pa- 


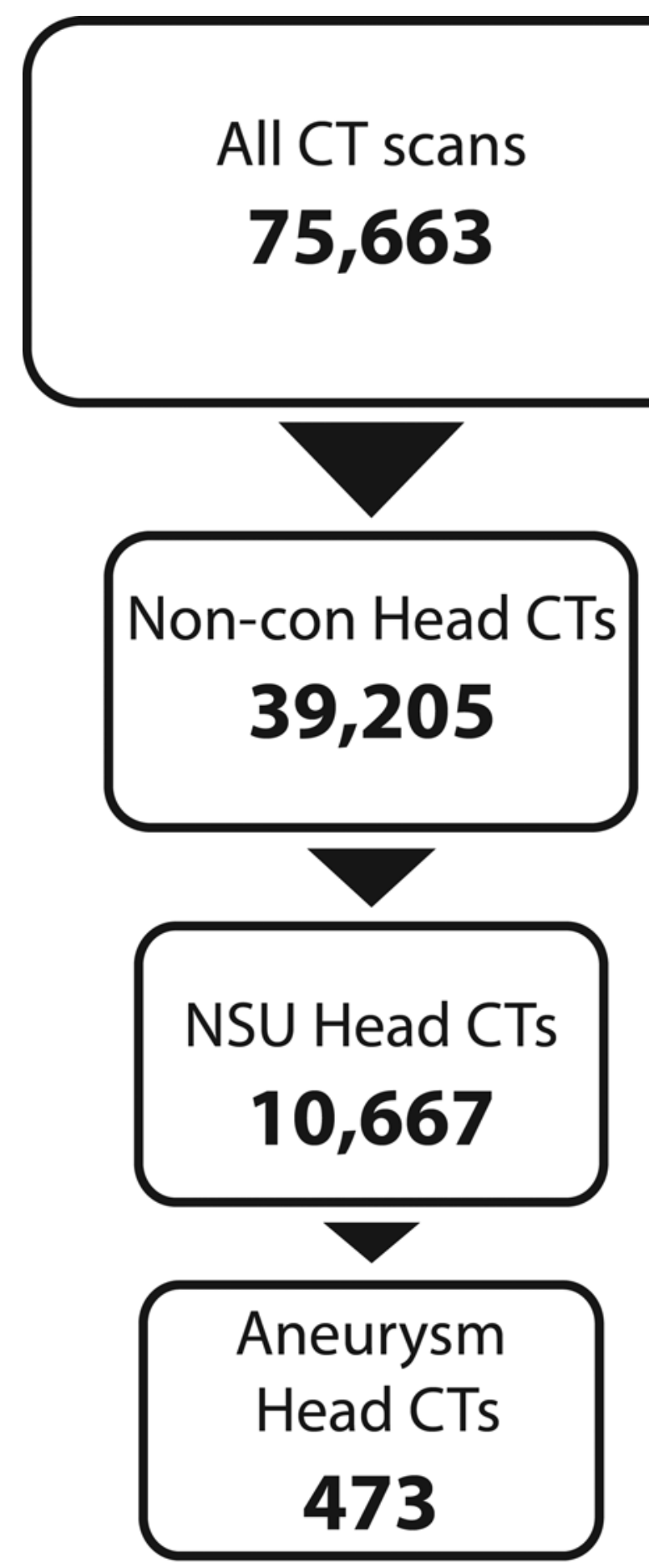

FIG. 1. Flow chart showing the number of CT scans and noncontrast head CT scans performed at our institution from January 2010 to December 2014. Non-con = noncontrast; NSU = neurosurgery providerordered scans.

tients with nonfocal neurological examinations or those who were neurologically intact, head CT affected management in only $4.8 \%$ and $1.1 \%$ of cases, respectively (Fig. 2). Specifically, for the 2 management changes in neurologically intact patients, 1 was administration of mannitol for postoperative edema, an intervention of uncertain
TABLE 1. Demographic and clinical characteristics of the patient cohort*

\begin{tabular}{|c|c|}
\hline Characteristic & Value \\
\hline Mean age in years & $60 \pm 15$ \\
\hline Female & $231(76)$ \\
\hline \multicolumn{2}{|l|}{ Aneurysm location } \\
\hline Anterior circulation & $206(68)$ \\
\hline Posterior circulation & $64(21)$ \\
\hline Both & $34(11)$ \\
\hline \multicolumn{2}{|l|}{ Surgical approach } \\
\hline Pterional craniotomy & $207(68)$ \\
\hline Orbitozygomatic craniotomy $\dagger$ & $62(20)$ \\
\hline Other & $35(12)$ \\
\hline \multicolumn{2}{|l|}{ Imaging, mean $\ddagger$} \\
\hline No. of CT scans & $1.54 \pm 1.0$ \\
\hline No. of MRI studies & $0.20 \pm 0.50$ \\
\hline No. of angiograms & $0.84 \pm 0.45$ \\
\hline \multicolumn{2}{|l|}{ Immediate postop neurological exam } \\
\hline Intact & $175(56)$ \\
\hline Nonfocal deficit & $42(14)$ \\
\hline Focal deficit & $78(26)$ \\
\hline Mean LOS in days & $9.3 \pm 7.2$ \\
\hline \multicolumn{2}{|c|}{$\begin{array}{l}\text { LOS = length of stay. } \\
{ }^{*} \text { Values indicate number of patients (\%) unless otherwise indicated. Means } \\
\text { are given with SDs. } \\
\dagger \text { Includes the orbitopterional approach. } \\
\ddagger \text { Imaging studies per patient over the course of the hospitalization for treat- } \\
\text { ment of cerebral aneurysm. }\end{array}$} \\
\hline
\end{tabular}

value. The second was a return to the OR for the evacuation of an asymptomatic epidural hematoma; however, one could debate whether the patient truly needed the evacuation as he was asymptomatic from the hematoma at the time of repeat surgery. Additionally, if the hematoma had become clinically important, the patient likely would have exhibited focal or nonfocal neurological signs prompting a CT scan and intervention.

Univariate analyses showed that the patient's postoperative neurological examination was statistically significantly associated with whether the CT scan affected clinical management ( $\chi^{2}$ test, $\mathrm{p}=0.0192$; Table 3 ). However, neither the patient's age nor aneurysm location (anterior vs posterior circulation vs both) were associated with the likelihood of the CT scan leading to a change in management ( $p>0.05$; Table 3). A mixed-direction multivariate stepwise regression model confirmed that the neurological examination was the only variable predictive of the clinical utility of the CT scan ( $\mathrm{p}=0.0595$; all other $\mathrm{p}$ values $>0.50$ ).

We then performed a cost analysis of our routine postoperative head CT use. The cost of a noncontrast head CT scan at our institution was $\$ 292$, and the mean cost per inpatient hospitalization for our nonruptured aneurysm patients was $\$ 72,227( \pm \$ 52,966)$. In our cohort, if a patient had a focal neurological deficit, 11 head CT scans were performed to obtain 1 head CT scan that served as the basis for altered management. This meant that $\$ 3212$ was 
TABLE 2. Changes in management resulting from head CT in all aneurysm patients

\begin{tabular}{lc}
\hline CT-Guided Management Changes & No. of Pts (\% of total pts) \\
\hline Blood pressure change & $4(1.3)$ \\
\hline Mannitol administration & $2(0.6)$ \\
\hline High-flow oxygen, lie flat & $2(0.6)$ \\
\hline Return to OR & $1(0.3)$ \\
\hline Other (Sz prophylaxis, Plavix dose) & $2(0.6)$ \\
\hline Total changes & $11(3.6)$ \\
\hline
\end{tabular}

Pts = patients; $S z$ = seizure.

spent to obtain 1 head CT scan responsible for changed management (Table 4), In contrast, for patients who were neurologically intact, 99 head CT scans were performed and $\$ 28,908$ was spent to obtain 1 head CT scan responsible for altered management (Table 4).

\section{Discussion}

Similar to prior studies, ${ }^{9,10}$ we find low rates of head CT scans used in altering the management of postoperative neurosurgical patients. Moreover, the clinical utility of head CT depends on the patient's neurological examination, with higher rates of positive findings on CT impacting management in patients with focal neurological deficits (9.0\% vs $4.8 \%$ and $1.1 \%$ in neurologically nonfocal and neurologically intact patients, respectively). Our findings likely represent an overestimate of true clinical utility for postoperative head CT in this patient population given the minimal impact or uncertain value of certain interventions (e.g., high-flow oxygen or mannitol administration) and our default option to attribute management changes to head CT if there was any uncertainty. Our rates of clinical utility are similar to those reported in the UCLA study, in which they found that only $1(<1 \%)$ of 126 postoperative CT scans served as the basis for changed management. ${ }^{10}$ Our intervention rates based on imaging findings are lower than those reported by Fontes et al., whose rates ranged from $2.2 \%$ to $22 \% .{ }^{9}$ This could reflect the more
TABLE 3. Univariate analysis of variables predicting whether CT scan leads to clinical management change

\begin{tabular}{lcl}
\hline \multicolumn{1}{c}{ Variable } & $\chi^{2}$ & $p$ Value \\
\hline Age & 0.020 & 0.894 \\
\hline Aneurysm location* & 0.832 & 0.660 \\
\hline Neurological exam $\dagger$ & 7.907 & $0.0192 \ddagger$ \\
\hline
\end{tabular}

\footnotetext{
* Anterior circulation, posterior circulation, or both.

$\dagger$ Intact, nonfocal, or focal deficit.

$\ddagger$ Indicates statistical significance at $p$ value $<0.05$.
}

heterogeneous patient population examined in their study, as they looked at all neurosurgical postoperative patients compared with only nonruptured aneurysm patients in our study.

Other so-called "screening" or "rule-out" examinations take place regularly in other areas of medicine, sometimes with minimal or nonspecific signs or symptoms, and with variable detection rates. For example, some providers and institutions aggressively seek to detect pulmonary embolism, leading to high utilization of CTPA (CT pulmonary angiography). In 1 study of emergency department, inpatient, and intensive care unit (ICU) patients at a single institution, the percentage of positive CTPA studies ranged from $15 \%$ to $17.9 \% .{ }^{6}$ In another study of postarthroplasty patients, however, the CTPA detection rate was as low as $2 \% .^{1}$ Overuse of CTPA leads not only to unnecessary radiation but also to possible overdiagnosis of disease, such as when nonspecialist physicians misinterpret artifacts or when a treated pulmonary embolism is too small or insignificant to actually cause the patient's symptoms. ${ }^{12}$ Attempts to decrease the use of CTPA using clinical algorithms have been met with limited success and emphasize that the need for methodologies to determine pretest probability for imaging studies is not isolated to neurosurgical cases.

Our cost analysis suggests that routine postoperative head CT may not be a good use of hospital resources in treating neurologically intact patients compared with patients with focal or nonfocal deficits on their postoperative examinations. It is important to emphasize, however, that

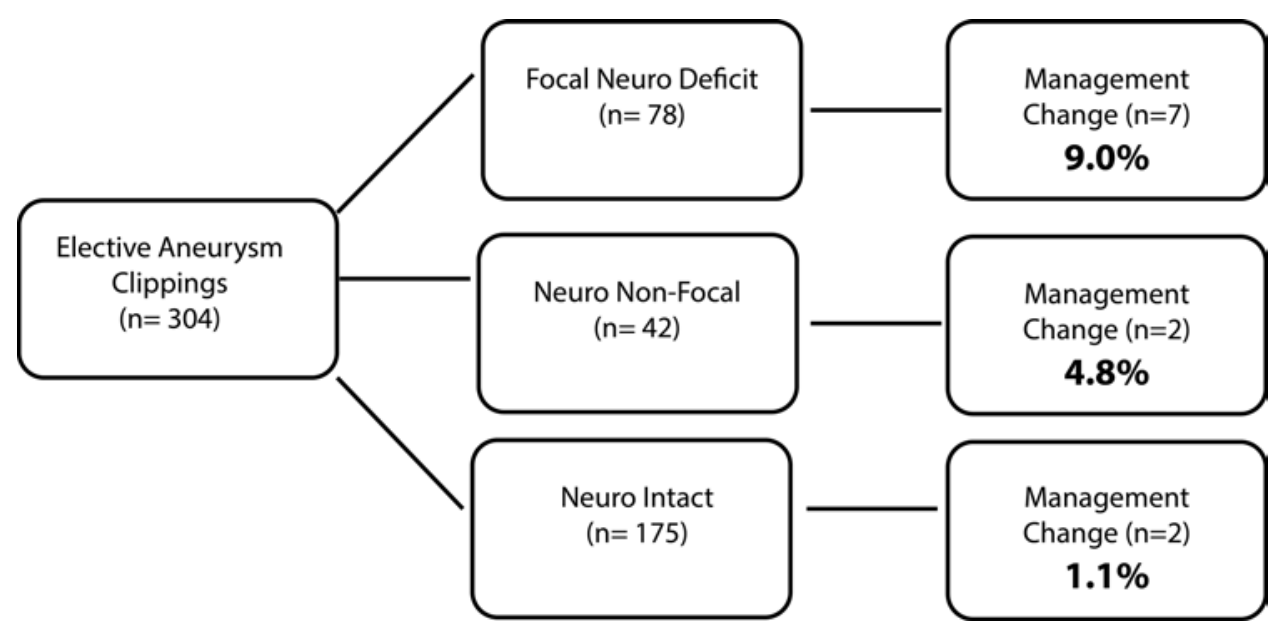

FIG. 2. Flow chart showing changes in treatment management in elective aneurysm surgical patients stratified by immediate postoperative neurological examination. Neuro = neurological(ly). 
TABLE 4. Number and cost of CT scans obtained in order to obtain 1 head image that led to changed management in patients with a nonruptured aneurysm

\begin{tabular}{ccc}
\hline Neurological Exam Findings & No. of CT Scans & Total Cost (US \$) \\
\hline Focal deficit & 11 & 3212 \\
\hline Nonfocal deficit & 20 & 5840 \\
\hline Intact & 99 & 28,908 \\
\hline
\end{tabular}

there are no standard accepted cutoffs in this area, such as, for example, the national thresholds for incremental costeffectiveness ratios. We therefore have to use our own clinical judgment, which suggests that, given the high number of scans (99) and dollars (\$28,908) needed to obtain 1 head CT scan that impacts clinical management in a small way (e.g., mannitol administration), we do not believe this to be a clinically useful intervention or good use of hospital resources for neurologically intact patients at our institution. There are obvious exceptions for individual cases, and physicians should always use their clinical judgment if they have concerns about a patient, particularly due to the potentially devastating consequences of postsurgical complications in neurosurgical patients. However, our data argue strongly in favor of the importance of the neurological examination, rather than turning to additional imaging that may not be clinically useful. We should note here that all aneurysm patients at our institution are admitted to the ICU postoperatively, where they undergo neurological examinations every hour by ICU nurses trained in the care of neurosurgical patients. Given the recent studies suggesting that not all postcraniotomy patients benefit from ICU-level interventions ${ }^{11}$ and the well-known high cost of ICU stays (\$5199 per day at our institution), it would be interesting to determine in a future study what subset of our nonruptured aneurysm patients truly require ICU stays, and whether routine head CT could help us to stratify these patients appropriately.

Another important consideration is that unnecessary CT scanning carries risk, as it exposes patients to small amounts of radiation and increases lifetime cancer risk. In our neurologically intact aneurysm patient population, to obtain 1 head CT scan that can lead to changes in clinical management, 99 patients are unnecessarily exposed to a cumulative 99 milliseivert $(\mathrm{mSv})$ of radiation (assuming $1 \mathrm{mSv}$ per head CT scan), with a total of $6.93 \%$ increased cancer risk across these patients (estimated based on Berrington et al. ${ }^{3}$ ). In contrast, for our postoperative patients with focal neurological deficits, to obtain 1 head CT scan that changes management, only 11 patients are exposed to a cumulative $11 \mathrm{mSv}$ of radiation, with a $0.77 \%$ increased cancer risk across these patients. ${ }^{3}$

An important limitation of our study is that this is a retrospective, single-site study. We acknowledge that a prospective evaluation of radiographic efficacy would allow us to better determine whether CT scans lead to changed clinical management, rather than trying to infer the decision-making process retrospectively from the medical record. Similarly, a multisite study with additional neurosurgical patient types could provide more generalizable data, although we purposefully chose to examine a single patient population at our institution for purposes of patient and clinical management homogeneity. Along these lines, there is well-documented variation in head CT use based on provider, and in this study, we looked at only 1 neurosurgical provider's practice..$^{15}$ As a result, it is important for us to caution that our findings (both in terms of CT yield and actionability of CT findings) may not be generalizable to other patient populations, surgeons, or institutions. Another important point is that we did not actually evaluate whether these management changes affected clinical outcome, which is difficult to determine, especially for interventions like seizure prophylaxis, where we cannot predict whether a patient would have had a seizure without the medication prophylaxis.

\section{Conclusions}

In summary, although there are no clear guidelines, the large number of CT scans and high cost of CT needed to treat neurologically intact elective aneurysm patients suggest that careful neurological monitoring may be more clinically useful and a better use of hospital resources than routine postoperative CT. On the basis of this study, we have altered our practice and no longer obtain routine postoperative head CT scans in neurologically intact patients after elective aneurysm clippings. Given the singlesite and single-surgeon nature of this study, we caution that our results may not be generalizable to other patient populations, surgeons, or institutions. Implementation of this type of policy should be studied at multiple institutions in multiple levels of care settings prior to consideration of such a strategy for an imaging efficiency measure.

\section{Acknowledgments}

Dr. Zygourakis received research funding from a UCSF Center for Healthcare Value Fellowship.

\section{References}

1. Allen C, Seinge R, Maxwell R, Thind D: CT pulmonary angiography and pulmonary embolism following 5809 primary joint arthroplasties. N Z Med J 128:41-49, 2015

2. Almenawer SA, Bogza I, Yarascavitch B, Sne N, Farrokhyar F, Murty N, et al: The value of scheduled repeat cranial computed tomography after mild head injury: single-center series and meta-analysis. Neurosurgery 72:56-64, 2013

3. Berrington de González A, Darby S: Risk of cancer from diagnostic X-rays: estimates for the UK and 14 other countries. Lancet 363:345-351, 2004

4. Brenner DJ: Should we be concerned about the rapid increase in CT usage? Rev Environ Health 25:63-68, 2010

5. Brown CV, Weng J, Oh D, Salim A, Kasotakis G, Demetriades D, et al: Does routine serial computed tomography of the head influence management of traumatic brain injury? A prospective evaluation. J Trauma 57:939-943, 2004

6. Costa AF, Basseri H, Sheikh A, Stiell I, Dennie C: The yield of CT pulmonary angiograms to exclude acute pulmonary embolism. Emerg Radiol 21:133-141, 2014

7. CostEvaluation.com: How much does a CT scan cost? (http:// www.costevaluation.com/ct-scan-cost.php) [Accessed online February 24, 2016]

8. Durham SR, Liu KC, Selden NR: Utility of serial computed tomography imaging in pediatric patients with head trauma. J Neurosurg 105 (5 Suppl):365-369, 2006 
9. Fontes RB, Smith AP, Muñoz LF, Byrne RW, Traynelis VC: Relevance of early head CT scans following neurosurgical procedures: an analysis of 892 intracranial procedures at Rush University Medical Center. J Neurosurg 121:307-312, 2014

10. Garrett MC, Bilgin-Freiert A, Bartels C, Everson R, Afsarmanesh N, Pouratian N: An evidence-based approach to the efficient use of computed tomography imaging in the neurosurgical patient. Neurosurgery 73:209-216, 2013

11. Hanak BW, Walcott BP, Nahed BV, Muzikansky A, Mian MK, Kimberly WT, et al: Postoperative intensive care unit requirements after elective craniotomy. World Neurosurg 81:165-172, 2014

12. Hutchinson BD, Navin P, Marom EM, Truong MT, Bruzzi JF: Overdiagnosis of pulmonary embolism by pulmonary CT angiography. AJR Am J Roentgenol 205:271-277, 2015

13. Kaups KL, Davis JW, Parks SN: Routinely repeated computed tomography after blunt head trauma: does it benefit patients? J Trauma 56:475-481, 2004

14. Klassen TP, Reed MH, Stiell IG, Nijssen-Jordan C, Tenenbein M, Joubert G, et al: Variation in utilization of computed tomography scanning for the investigation of minor head trauma in children: a Canadian experience. Acad Emerg Med 7:739-744, 2000

15. Prevedello LM, Raja AS, Zane RD, Sodickson A, Lipsitz S, Schneider L, et al: Variation in use of head computed tomography by emergency physicians. Am J Med 125:356-364, 2012

16. Schunk JE, Rodgerson JD, Woodward GA: The utility of head computed tomographic scanning in pediatric patients with normal neurologic examination in the emergency department. Pediatr Emerg Care 12:160-165, 1996

17. Sifri ZC, Homnick AT, Vaynman A, Lavery R, Liao W, Mohr $A$, et al: A prospective evaluation of the value of repeat cranial computed tomography in patients with minimal head injury and an intracranial bleed. J Trauma 61:862-867, 2006

18. Sifri ZC, Livingston DH, Lavery RF, Homnick AT, Mosenthal AC, Mohr AM, et al: Value of repeat cranial computed axial tomography scanning in patients with minimal head injury. Am J Surg 187:338-342, 2004

\section{Disclosures}

Dr. Lawton reports being a consultant for Zeiss and receiving royalties from Mizuho America.

\section{Author Contributions}

Conception and design: Lawton, Zygourakis, Pitts, Hannegan. Acquisition of data: Zygourakis, Winkler, Franc. Analysis and interpretation of data: Lawton, Zygourakis, Winkler. Drafting the article: Zygourakis. Critically revising the article: Lawton, Zygourakis, Pitts, Hannegan, Franc. Reviewed submitted version of manuscript: Lawton, Zygourakis, Winkler, Franc. Approved the final version of the manuscript on behalf of all authors: Lawton. Statistical analysis: Zygourakis. Study supervision: Lawton.

\section{Supplemental Information}

\section{Previous Presentations}

Portions of this paper were presented in 2015 at the Annual Meeting of the American Association of Neurological Surgeons (May 2-6, Washington, DC).

\section{Correspondence}

Michael Lawton, Department of Neurological Surgery, University of California, San Francisco, 505 Parnassus Ave., Rm. M779, San Francisco, CA 94143. email: lawtonm@neurosurg.ucsf.edu. 